\title{
Emotional Stress: The Dialectics of Nature
}

\section{Evgeny Antonovich Yumatov}

P.K. Anokhin Scientific Research Institute of Normal Physiology, Moscow, Russia

Email: eayumatov@mail.ru

How to cite this paper: Yumatov, E.A. (2020) Emotional Stress: The Dialectics of Nature. Neuroscience \& Medicine, 11, 20-28. https://doi.org/10.4236/nm.2020.111003

Received: January 27, 2020

Accepted: February 28, 2020

Published: March 3, 2020

Copyright (c) 2020 by author(s) and Scientific Research Publishing Inc. This work is licensed under the Creative Commons Attribution International License (CC BY 4.0).

http://creativecommons.org/licenses/by/4.0/

\begin{abstract}
The article considers the biological nature and origins of emotional stress. Emotional stress is primarily formed within the mental activity of the brain in the form of pronounced negative emotions. The "Dynamic Theory of Emotions" characterizes the development of a continuous negative emotional state in conflicting behavioral situations when the subject is unable to satisfy its strong dominant need for a long time. The emotional stress is represented as having a dual nature, i.e. a biologically negative pathogenetic effect on health, but a positive impact on the adaptation of individuals, self-preservation of life, and evolutionary change in species.
\end{abstract}

\section{Keywords}

Emotions, Emotional Stress, Psychosomatic Diseases, Health, Adaptation, Evolution

\section{Introduction}

The concept of stress as a general non-specific adaptive syndrome of the body was first formulated in the works of H. Selye [1] [2] [3]. According to H. Selye's definition, stress is a reaction that arises as a nonspecific response of an organism to the action of extreme, adverse environmental factors, or "stressors", that are pathogenic agents, toxic and foreign substances, physical factors, and other inappropriate influences. According to its biological purpose, stress has an adaptive focus, and activates protective mechanisms to prevent the pathogenic effect of adverse factors on the body.

Stress is characterized by a dual nature that is adaptive and pathogenetic. Stress develops through a series of successive phases: anxiety, resistance, and exhaustion. The body reacts differently to stressful effects in different phases of stress. In the phase of anxiety, primary stress reactions appear. In the phase of resistance, the body enhances its protective adaptive functions that contribute to 
overcoming an unfavorable situation; in the phase of exhaustion, the stress state has a negative damaging effect on the physiological functions of the body. The last stage may end with the death of the body.

The stress reaction in all cases is formed due to the activation of the pituitary-adrenal mechanisms, including the activation of ACTH and adrenocorticoid function of the adrenal glands [2] [3].

Along with this, science has developed an idea of emotional stress as a psychoemotional state of a subject, which is characterized by a complex of nonspecific (with respect to initiating emotional factors) psychophysiological, autonomic, and hormonal manifestations [4]-[9].

\section{The Origin of Emotional Stress}

Primary emotional stress is formed in the mental activity of the brain as pronounced, prolonged negative emotions arising in a conflict behavioral situation [4] [5] [7] [8] [9] [10].

Systematic dissatisfaction with the results of behavior, associated with the lack of the subject's ability to achieve an adaptive result, generates a continuous negative emotional stress.

The "Dynamic Theory of Emotions" formulated by us describes the sequential development of positive and negative emotions at different stages of purposeful behavior, taking into account the changing relationships of predicted probability and real achievement of the result, as well as individual characterological personality traits [11] [12].

The "Dynamic Theory of Emotions" characterizes the appearance of a continuous negative emotional state in conflicting behavioral situations when the subject is unable to satisfy its strong dominant need for a long time.

You can define the concept of emotional stress as a complex of psycho-emotional and somatovegetative reactions of the body that arise in a conflict behavioral situation when humans and animals cannot satisfy their leading biological or social needs [10].

The development of emotional stress depends on the individual subjective perception of the proneness to conflict in the prevailing behavioral situation. A conflict situation arises only when the individual perceives it in this way.

In our experiments, it was shown that rats forced to stay in tight cases, under conditions of immobilization, experienced emotional stress. However, if the rats voluntarily climbed in and stayed in the same cases within the same time, fleeing from electrodermal impact, then they did not experience any emotional stress. The physical condition of rats in these two situations was the same, but their subjective attitude to their position was different, and this explains the fact that when the rats stayed in the cases of their own free will, as if in a burrow, they did not experience emotional stress [13].

It should be noted that with full satisfaction of all the needs and vagaries of the individual, a negative emotional state sets in, characterized by emotional "sa- 
tiety", when nothing pleases, and life seems empty. Only if there is a longing for a passionate expectation of the desired result will a positive emotion manifest itself when this result is achieved [11] [12] [14].

The causes of emotional stress are:

- Social conflicts: war, overpopulation, poverty, hunger, limited food and water resources, the criminal environment, crime.

- Socio-political conflicts: revolutions, social injustice, political instability.

- Environmental disasters: earthquakes, volcanic eruptions, tsunamis, floods, fires, adverse environmental conditions, etc.

- Domestic and industrial conflicts: information overload, excessive work, overwork, moral dissatisfaction, interpersonal competition, humiliation, insult, violence, poor living conditions, personal or family conflicts, poverty, death and loss of loved ones, loneliness, isolation, unrequited love, jealousy, envy, restriction of freedom, illness, satiety with material goods, etc. [15] [16].

It is unlikely that humanity will ever solve all these problems and avoid emotional stress. Causes and conditions for emotional stress will always exist in human society and in nature. Emotional stress is inevitable in life. Moreover, it can have both a positive influence on humanity and a negative impact on the life and health of people. The question is, what are the detrimental effects of emotional stress, and what is its positive impact?

\section{Biologically Negative Role of Emotional Stress}

Emotional stress, as well as stress (according to H. Selye), is nonspecific in relation to any kind of conflict situation complex of reactions affecting vital physiological functions [12] [17] [18].

Emotional stress may be the cause of many psychosomatic diseases: psychosis, neurosis, sleep disturbances; cardiovascular diseases - arrhythmias, extrasystoles, myocardial infarction, hypertension; ulcerative-dystrophic lesions of the gastrointestinal tract; decreased immunity and increased susceptibility to viral and infectious diseases; autoimmune processes; rheumatic diseases, osteochondrosis; oncological diseases; hormonal and sexual dysfunctions, etc. [4] [5] [9] [19] [20].

Emotional stress may cause serious psychoneurotic reactions [16] [21]. It was noted that individuals exhibit excessive emotionality, irritability, excessive suspiciousness, anxiety, suspicion, a tendency to prolonged emotional experiences; sleep is disturbed, disability is reduced, and memory is impaired. Emotional reactions lose their plasticity and cease to be adequate factors in mobilizing behavior to satisfy a specific adaptive result.

Stress affects the genetic apparatus of cells, leading to congenital disorders in the children's development and health. The detrimental effect of stress is manifested in the growth of alcoholism and drug addiction, in the increase in injuries, in the increase in the number of suicides, in the incapacitation of society. Emotional stress is the main reason for reducing life expectancy, increasing mortality 
and, in particular, sudden death [14] [15] [16] [22].

Emotional stress primarily arises as a central neurogenic process, while all peripheral disturbances of vital somatovegetative functions develop secondarily and are actually the result of negative emotional excitation [10] [12].

During emotional stress in the emotional structures of the brain, changes occur in the content of the main mediators such as norepinephrine, acetylcholine, serotonin. The most pronounced change in catecholamine content during emotional stress occurs in the hypothalamus and is characterized by a decrease in norepinephrine and an increase in dopamine content [13] [23].

In the effector realization of emotional excitations, the main role is played by hormonal mechanisms, and primarily the pituitary-adrenal system [8] [19] [24]. Emotional excitation is characterized by certain hormonal reactions: an increase in the concentration of catecholamines in the blood (adrenaline, norepinephrine, dopamine), due to their release by the adrenal glands, an increase in the secretion of thyroid hormones, an increase in the level of cyclic AMP, prostaglandins and renin activity in blood plasma.

With emotional stress, selective disturbances of various physiological functions may occur: cardiovascular, gastrointestinal, etc. Against the background of the stability of some physiological functions, disturbances of others may occur. For instance, during in our studies, we saw the formation of ulcers of the stomach wall under emotional stress wherein the cardiovascular functions were stable [9] [25].

Thus, emotional stress is a systemic multi-level reaction of the body to a conflict situation [26].

The presence of social programs, the upbringing of a social culture, and the development of healthcare aimed at reducing emotional stress and preventing physiological functions caused by stress will not be able to completely eliminate emotional stress. However, this does not mean that it is not necessary to prevent emotional stress, and to prevent its detrimental effect on the life and health of people [16] [27].

\section{Biologically Positive Role of Emotional Stress}

When studying emotional stress, the average method for analyzing the manifestations of emotional stress is used, which does not allow us to identify individual-group differences in the body's reaction to a stressful situation.

In our studies, when studying emotional stress, we used the method of individual analysis of the physiological reactions of individual animals, which allowed us to identify the mechanisms of resistance and adaptation to stress that certain animals possessed [28].

As it turned out, in conflicting behavioral situations, different individuals exhibit different resistance to emotional stress, which is characterized by maintaining the stability of vital functions of the body [13] [19].

Resistance to emotional stress is determined by the severity of the classic ma- 
nifestations of stress. Among them there are indicators of adrenal hypertrophy, thymus involution, ulcerative and dystrophic disorders in the stomach, changes in the content of catecholamines in the emotiogenic structures of the brain, as well as cardiovascular parameters. The most general criterion characterizing the resistance of animals to emotional stress is the indicator of animal survival in conflicting behavioral situations.

A characteristic central sign of emotional stress is a decrease in the content of noradrenaline in the hypothalamus. The increase and normalization of the amount of noradrenaline in the hypothalamus correlates with resistance to emotional stress and may be considered as one of the key factors for resistance to it [13] [26].

It has been established that rats that are resistant and susceptible to emotional stress manifest selective changes in biogenic amines in the emotiogenic brain structures [3].

Resistance to emotional stress depends on genetic and individual development factors. In our experiments, it was shown that in the conflict situations of the same type, animals of different pure lines and nonlinear animals exhibit different individual resistance to emotional stress [13] [26] [29].

One can observe adaptation to chronic stressful effects, in which the body passes from a pathogenic reaction to an adaptation state.

An important condition for adaptation during emotional stress is the body's ability to restore normal levels of noradrenaline in the hypothalamus, increase its content in the midbrain, and also increase dopamine levels in the hypothalamus, midbrain and medulla oblongata [23].

The positive role of emotional stress is manifested in its adaptive capabilities.

The formation of stability and adaptation to a stressful situation is a positive factor in emotional stress.

The adaptive role of emotional stress helps overcome obstacles to achieving a useful adaptive result, and self-preservation of the body in conflict behavioral situations.

Adaptation during emotional stress in a person can occur at the level of mental, conscious brain activity, in which a person finds adequate behavioral ways to solve or avoid a conflicting behavioral situation [12] [27].

The sequential development of phases of emotional stress in individuals does not occur the same way. An individuality is observed, which depends on many factors: on the severity of the conflict situation, on the subjective perception of its significance, on the characterological traits of the person, on the degree of development of emotional stress, on individual resistance to stress, and on the ability to adapt.

In the same conflict situation, it can be seen that in some individuals the stress will be limited to the adaptation phase, while in others the phase of exhaustion will appear.

In different phases of emotional stress, the hypothalamic-pituitary-adrenal system functions differently. The activity and physiological effects of stress hor- 
mones will be different, depending on what phase of stress is currently taking place in the body. Stress hormones not only cause a stress-damaging effect, but can also have a stress-protective effect; particularly, glucocorticoids prevent ulceration in the stomach during immobilizational emotional stress [24].

The positive role of emotional stress is manifested in the activation of creative abilities in various human activities. The stimulus to creativity is often social, moral, domestic dissatisfaction. Often such a stimulus takes form of unrequited, unhappy love. An ideal, comfortable, materially excessive environment, as a rule, does not contribute to creative activity. To one degree or another, creativity is always associated with emotional stress.

A discussion is underway, is the evolutionary development of man and animals still happening or stopped [30].

As you know, biological evolution is a natural process of development of wildlife, accompanied by a change in the genetic composition of populations, the formation of adaptations, speciation and extinction of species, the transformation of ecosystems and the biosphere as a whole [31].

J. Pickrell et al. found evidence that the "Darwin" natural selection in humanity is currently taking place. Large-scale genetic analysis of the DNA of 160 thousand inhabitants of Great Britain and the USA shows that the biological evolution of mankind has not stopped and that the number of carriers of "harmful" versions of the genes associated with diseases continues to fall gradually under the influence of natural selection [30].

We can assume that the evolutionary process continues, but at present other factors are involved in it, related to the development of civilization, industry, with the growth of the Earth's population, with the change in the ecological environment of human and animal life, with the extinction of a number of species, with the existence of emotional stress.

In this regard, let us consider the role of emotional stress in biological evolution. In this case, we will focus not so much on the survival of the species as on its modification.

During overpopulation of animals, lack of food resources, development of emotional stress, increase in aggressiveness, and cannibalism are observed, causing a limitation in the number of species, which, ultimately, in an unfavorable ecological environment leads to the extinction of the species, and to interspecies imbalance. This inevitably affects the survival of all other related species.

With emotional stress, selective survival of adaptive individuals in the population occurs. Persons prone to emotional stress are eliminated. Thus, emotional stress carries out natural selection, which affects population stability and the evolution of the species.

Emotional stress leads to infertility, a decrease in potency and reproductive functions, and in some cases death from cardiovascular and oncological diseases of young people at adulthood. As a result, elimination of unstable and non-adaptable sexually mature individuals is observed, with simultaneous adap- 
tation to the emotional overstrain of more stable individuals. This happens in each subsequent generation, which causes a gradual evolutionary modification. Obviously, the effect of emotional stress on older people of non-childbearing age is less significant for the evolutionary process.

Existing social programs and modern health care have limited opportunities to compensate for negative processes caused by emotional stress, and therefore cannot significantly affect the evolutionary role of emotional stress [16] [27].

Due to emotional stress, biological self-regulation of the species abundance and its evolutionary change through natural selection, and the preservation of individuals most resistant to emotional stress, occurs.

Along with stress, the evolution of the human species is affected by demographic mixing of races, modification of the population, extinction of certain animal species and the related redistribution of all interspecific relationships and their survival conditions-all that leads to the elimination of individuals not adapted to modern conditions.

Thus, emotional stress is one of the factors in the modern evolution of species.

As you know, evolution took place over millions of years. The current evolution is not visible due to the short duration of the observation period available to us.

\section{Conclusions}

Emotional stress arose as a result of biological patterns of nature. At its core, emotional stress is inevitable, and it cannot be completely excluded from the social and biological organization of life.

At the same time, emotional stress reflects the dialectics of nature and has two opposite properties: positive and negative.

Undoubtedly, a negative manifestation of stress has a detrimental effect on the vital functions of the body, leading to disease, early aging and often death. Medical social programs are directed against the existence of this negative effect of emotional stress on the life and health of people.

However, emotional stress has an important positive general biological, social and evolutionary role, aimed at adaptation, increasing the stability of individuals and the species as a whole, adaptation to constantly changing living conditions.

This general biological role of emotional stress arises from its main property-to increase at a certain stage of its development the adaptive capabilities of the body, which are not the same in all individuals.

In an individual analysis of emotional stress, we found that individual animals exhibit different resistance to emotional stress and the selectivity of disorders of different physiological functions, which depend on genetic and individual development factors. The stability of physiological functions during emotional stress is formed as a result of individual adaptation and in the evolutionary process. 
The formation of stability and adaptation to a stressful situation is a positive factor in emotional stress in the evolutionary process of survival and conservation of species.

Due to emotional stress, biological self-regulation of the species abundance occurs and its evolutionary change happens through natural selection, due to the self-preservation of individuals most resistant to emotional stress and elimination, predisposed to emotional stress of individuals.

\section{Conflicts of Interest}

The author declares no conflicts of interest regarding the publication of this paper.

\section{References}

[1] Selye, H. (1979) Stress without Distress. “Progress”, M., 123 p. (Russian)

[2] Selye, H. (2008) Stress of Life. M. (Russian)

[3] Selye, H. (1956) The Stress of Life. McGraw-Hill, New York, 324 p.

[4] Anokhin, P.K. (1965) Emotional Stress as a Prerequisite for the Development of Neurogenic Diseases of the Cardiovascular System. Vest of USSR Academy of Medical Sciences, 20, 10-18. (Russian)

[5] Gelgorn, E. and Luffborough, J. (1966) Emotions and Emotional Upsets. M. (Russian)

[6] Levi, L. (1967) Emotional Stress. S. Karger. Basel, 542 p.

[7] Levi, L. (1970) Emotional Stress. Medicine, M., 329 p. (Russian)

[8] Levi, L. (1972) Stress and Distress in Response to Psychosocial Stimuli. Oxford Pergamon Press, Oxford, 480 p.

[9] Levi, L. and Kagan, A. (1980) Psychosocially Induced Stress and Disease. In: Selye, H., Ed., Problem Research Strategies and Results. Guide to Stress Research, von Nostrand Reinhold Co., New York, 118-130.

[10] Sudakov, K.V. (1981) Systemic Mechanisms of Emotional Stress. M. Medicine, 229 p. (Russian)

[11] Yumatov, E.A. (2009) Dynamic Organization of Emotions and Emotional Stress. Materials of the 6th Simonov Readings, 13-46. (Russian)

[12] Yumatov, E.A. (2019) Dynamic Theory of Emotions and Systematic Organization of Behavior. Herald of the International Academy of Sciences, No. 1, 56-65. (Russian) http://www.heraldrsias.ru/journals/2019/1/407/ http://www.heraldrsias.ru/download/articles/10_Yumatov.pdf

[13] Yumatov, E.A., et al. (1997) Emotional Stress: Theoretical and Clinical Aspects. Committee on Press and Information. 168 p. (Russian)

[14] Simonov, P.V. (1970) Theory of Reflection and Psychophysiology of Emotions. M. (Russian)

[15] Sudakov, K.V. and Yumatov, E.A. (1991) Emotional Stress in Contemporary Life. M. Publishing House of NPO Soyuzmed Inform, 81. (Russian)

[16] Yumatov, E.A. (2011) Chap. 3, p. 71, Emotional Stress; Ch. 4, p. 103, Practical Aspects of the Study and Prevention of Emotional Stress; Ch. 5, p. 133, Socio-Economic Background for the Development of Emotional Stress. In the Guide: 
Emergency Psychiatry, 2nd Edition, Revised and Supplemented, in 2 Volumes. Edited by prof. Z.I. Kekelidze. M., GUZ Regional Psychiatric Hospital of the Ministry of Health of the Khabarovsk Territory, Vol. 1. (Russian)

[17] Lazarus, R.S. (2012) Theory of Stress and Psychophysiological Studies. In: Levy, L., Ed., Emotional Stress, L. Medicine. (Russian)

[18] Yumatov, E.A., Glazachev, O.S., Bykova, E.V., Dudnik, E.N., Potapova, O.V. and Pertsov, S.S. (2017) Psychophysiology of Emotions and Emotional Stress of Students. M. ITRK, 200 p. (Russian)

[19] Korneva, E.A. and Shkhinek, E.K. (1984) Hormonal Stress Components and Protective Functions of the Body. Emotions and Behavior: A Systematic Approach. M., 155 p. (Russian)

[20] Chazov, E.I. (1975) Emotional Stress and Cardiovascular Disease. Vest USSR Academy of Medical Sciences, No. 8, 3-8. (Russian)

[21] Ayrapetyants, M.G. and Wayne, A.M. (1982) Neurosis in the Experiment and in the Clinic. M., Publishing House "Science". (Russian)

[22] Sudakov, K.V. and Yumatov, E.A. (1980) Acute Emotional Stress as a Cause of Sudden Death. In: Wichert, A.M. and Laun, B., Ed., Sudden Death, M. Medicine, 360-368. (Russian)

[23] Anokhina, I.P., Ivanova, T.M., Skocelyas, Y.G. and Yumatov, E.A. (1985) The Content of Biogenic Amines in Various Brain Structures in Rats Adapted to Chronic Emotional Stress. M. of Higher Nervous Activity, No. 2, 348-353. (Russian)

[24] Filaretova, L.P. (2010) Stress in Physiological Studies. I.M. Sechenov Russian Physiological Journal, 96, 924-935. (Russian)

[25] Krokhina, E.M., Skocelyas, Y.G. and Yumatov, E.A. (1977) Disorders of the Adrenergic Innervation of the Stomach in Rats with Stable Regulation of Cardiovascular Functions during Emotional Stress. Bulletin of Experimental Biology and Medicine, 84, 505-507. (Russian) https://doi.org/10.1007/BF00801140

[26] Yumatov, E.A. (1982) A Systematic Approach as a Conceptual Basis for the Study of Emotional Stress. Herald of the Academy of Medical Sciences of the USSR, No. 2, 63-69. (Russian)

[27] Yumatov, E.A. (2010) Social Culture as the Basis of the Spiritual, Moral and Economic Progress of Russia. Herald of the Russian Section of the International Academy of Sciences. (Russian)

[28] Yumatov, E.A. and Skocelyas, Y.G. (1979) Comparative Analysis of the Stability of Cardiovascular Activity in Different Rat Strains under Experimental Emotional Stress. Higher Nervous Activity, 29, 345-352. (Russian)

[29] Krockina, E.M., Skocelias, Y.G. and Yumatov, E.A. (1979) Functional State of Adrenergic Innervation of the Stomach in Rate with Different Stability of Cardiovascular Functions in Experimental Stress. Annales d'Anetômie pathologique, 24, 3-14.

[30] Mostafavi, H., Berisa, T., Day, F.R., Perry, J.R., Przeworski, M. and Pickrell, J.K. (2017) Identifying Genetic Variants that Affect Viability in Large Cohorts. PLoS Biology, 15, e2002458. https://journals.plos.org/plosbiology/article?id=10.1371/journal.pbio.2002458

[31] Darwin, C. (2001) On the Expression of Emotions in Humans and Animals. St. Petersburg, 384 p. (Russian) 